\title{
Treatment and prognosis of pyogenic liver abscess
}

\author{
Yu-Jang Su • Yen-Chun Lai • Yu-Chia Lin • Yu-Hang Yeh
}

Received: 14 December 2009 / Accepted: 24 August 2010/Published online: 5 November 2010

(C) The Author(s) 2010. This article is published with open access at Springerlink.com

\begin{abstract}
Background There are many combinations of treatments for pyogenic liver abscess (PLA). Different treatments are indicated for different clinical courses of PLA.

Aims To realize the current prevalence, clinical course, trend of treatment, and prognosis of pyogenic liver abscess (PLA) in Taiwan.

Methods We retrospectively reviewed the medical records of 126 patients with PLA. We divided them into four groups: M, $\mathrm{MD}, \mathrm{MS}$, and MDS, represented as parenteral antibiotic only, parenteral antibiotic with drainage, parenteral antibiotic with surgical intervention, and parenteral antibiotic with surgical drainage. We analyzed data by commercial statistical software (SPSS for Windows, version 11.0, SPSS Ltd., Chicago, IL). We used Student's t-test and $\chi^{2}$ test for statistical analyses, and significance was set at a $p$ value less than 0.05 .

Results PLA patients who were treated only with parenteral antibiotics had early diagnosis of PLA with a mean fever

\section{Y.-J. Su $(\bowtie) \cdot$ Y.-H. Yeh}

Department of Emergency Medicine, Mackay Memorial Hospital,

No.92, Sec 2 Chung-Shan North Rd,

Taipei 10449, Taiwan

e-mail: yjsu@ms1.mmh.org.tw

\section{Y.-J. Su}

Mackay Medicine, Nursing and Management College,

No.92, Shengjing Rd.,Beitou District,

Taipei 112, Taiwan

\section{Y.-C. Lai}

Department of Anesthesiology, Wan Fang Hospital,

Taipei Medical University,

Taipei 116, Taiwan

\section{Y.-C. Lin}

Department of Family Medicine, Mackay Memorial Hospital, No.92, Sec 2,Chung-Shan North Rd,

Taipei 10449, Taiwan
\end{abstract}

period of 3.3 days $(p=0.043)$. Patients who needed surgical drainage were highly associated with shock presentation in the clinical course $(35.7 \%$ versus $14.3 \%, \mathrm{p}=0.007)$.

Conclusion The earlier we can diagnose PLA, the shorter the patient's hospital stay (20.6 days) will be. In PLA patients with shock, a higher rate of surgical intervention is mandatory to save their lives.

Keywords Pyogenic liver abscess $\cdot$ Fever $\cdot$ Abdominal pain $\cdot$ Shock $\cdot$ Drainage

\section{Introduction}

Pyogenic liver abscess (PLA) is not a rarely seen disease in the emergency department (ED). It was sometimes diagnosed as flu initially owing to non-specific symptoms, such as fever, poor appetite, abdominal pain, and diarrhea. We can use bedside ultrasound for the early detection of liver abscess when we suspect it in someone who has flu-like symptoms, shaking chills, mild right upper quadrant discomfort, or who has risk factors, such as diabetes. We want to determine the optimal treatment of choice for the different kinds of clinical course of PLA. In the meantime, we also analyzed the general appearance of PLA, basic data, decision making in treatment, prognosis, and outcome. We can predict the optimal kind of treatment and explain this to the patients who are diagnosed with PLA in the ED.

\section{Materials and methods}

In a retrospective chart review from 1 January 2007 to 30 June 2008, there were 140 patients with liver abscesses hospitalized in a medical center in northern Taiwan. All the 
PLA patients were diagnosed by ultrasound or computed tomography. In our study, we also recorded the duration of fever until the PLA was diagnosed. In order to reduce the confounding factors, patients who were transferred from another hospital or had amebic, fungal, traumatic, or iatrogenic PLA were excluded because of the possibility of influencing the natural course of PLA. So we enrolled 126 patients into our study.

The 126 PLA patients ranged from 20 to 93 years old with a mean \pm SD of $56.5 \pm 15.7$. We collected data on clinical manifestations, laboratory data, images (abdominal sonography and/or computer tomography), blood and pus cultures, operation findings, and length of stay for statistical analyses. Symptoms were described as being present at the emergency or outpatient department visit. Shock was defined as systolic blood pressure (SBP) less than $90 \mathrm{mmHg}$ at the initial presentation. We also recorded the days of fever on arrival at the hospital (DFA) to determine how early the PLA case had been diagnosed.

We divided them into four groups according to the method of treatment: M, MD, MS, and MDS, each represented as parenteral antibiotic, parenteral antibiotic with drainage, parenteral antibiotic with surgical intervention, and parenteral antibiotic with surgical intervention and surgical drainage. We analyzed data by commercial statistical software (SPSS for Windows, Version 11.0, SPSS Ltd., Chicago, IL). We used Student's t-test and $\chi^{2}$ test for statistical analyses, and the significance was set at a $p$ value less than 0.05 .

\section{Results}

The enrolled 126 PLA patients had fever $(84.9 \%, \mathrm{n}=107)$, abdominal pain $(50.8 \%, \mathrm{n}=64)$, diarrhea $(11.1 \%, \mathrm{n}=14)$, history of diabetes $(42.1 \%, \mathrm{n}=53)$, and shock (Fig. 1) at presentation. Upon arrival at our hospital, they had fever ranging from 0 to 30 days with a mean $\pm \mathrm{SD}$ of $4.7 \pm$ 5.9 days. In the laboratory data, the white blood cell count

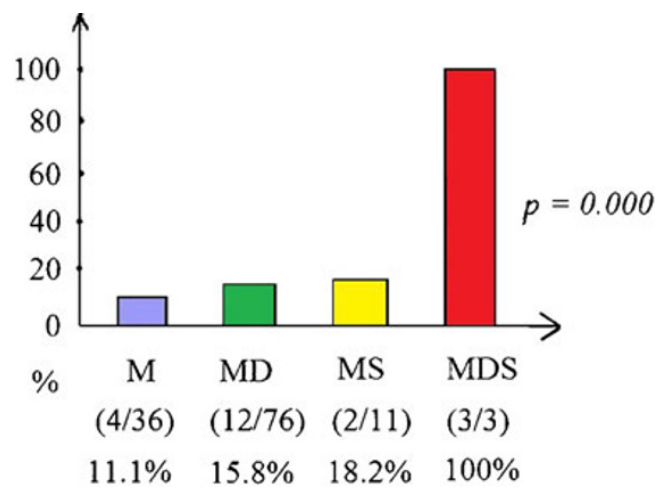

Fig. 1 Percentage cases of shock in the four groups of PLA cases
(WBC) ranged from 900 to $38,800 / \mu 1$ with a mean \pm SD of $14,760 \pm 6,962 / \mu 1$. The overall length of hospital stay was $24.2 \pm 13.9$ days. Five patients $(4 \%)$ died in the hospital (Table 1). The prevalence of bacteriology is shown in Table 2, and the mortality rate in four groups is shown in Table 3.

The majority $(60.3 \%)$ of patients with PLA were treated with antibiotics with drainage. We can see that the PLA patients needing surgical intervention (MS and MDS groups) had higher rates of shock presentation $(p=0.000$; Fig. 1). There was a significantly higher rate of shock presentation in PLA patients needing surgical interventions (35.7\% versus $14.3 \%, p=0.007)$. The PLA patients with shock had a significantly longer hospital stay (LOS) than the PLA ones without shock $(31 \pm 21.3$ versus $22.9 \pm$ 11.6 days, $p=0.01$; Fig. 2). On the other hand, the group with medical treatment had significantly shorter hospital stays (20.6 \pm 9.5 days, $p=0.029$; Fig. 3 ).

\section{Discussion}

Pyogenic liver abscess (PLA) is not an uncommon medical emergency involving all ages from the young to the very old. It could be caused by infection of biliary, portal, hematogenous, or cryptogenic origin and infection of adjacent structures [1]. The initial symptoms might be flulike, such as fever, abdominal pain, anorexia, nausea, mild diarrhea, or dizziness. In our study, $15.1 \%$ of PLA patients had no fever at presentation initially. This is the reason why PLA sometimes was found late. According to our study, it is commonly seen in middle aged men with a mean age of 56.5 years. The male-to-female ratio is $1.74: 1$, and the mean age of men who got PLA is lower than that in women.

Concerning the symptoms of PLA, only half of the patients who had abdominal pain, while $11.1 \%$ had diarrhea. Because the symptoms of PLA are sometimes subtle, delay before the diagnose PLA is seen occasionally in clinical practice. In our study, the mean time of fever in the four groups, M, MD, MS, and MDS, are 3.3, 5, 6.5, and

Table 1 Gender and symptoms of the 126 pyogenic liver abscess (PLA) cases

\begin{tabular}{lllll}
\hline & $\mathrm{n},(\%)$ & & $\mathrm{n},(\%)$ & $P$ value \\
\hline Male & $80(63.5 \%)$ & Female & $46(36.5 \%)$ & \\
Age(years) & $53.9 \pm 16.1$ & Age (years) & $60.9 \pm 14.1$ & $P=0.000$ \\
Abdominal pain & $64(50.8 \%)$ & & & \\
Fever & $107(84.9 \%)$ & & & \\
Diarrhea & $14(11.1 \%)$ & & & \\
Diabetes & $53(42.1 \%)$ & & & \\
Shock & $21(16.7 \%)$ & & & \\
\hline
\end{tabular}


Table 2 The results of pus cultures and blood cultures of PLA patients

\begin{tabular}{ll}
\hline Pus culture, $\mathrm{n}=88 . \mathrm{n}(\%)$ & Blood culture, $\mathrm{n}=121 ; \mathrm{n}(\%)$ \\
\hline No growth $20(22.7 \%)$ & No growth $57(46.7 \%)$ \\
Klebsiella pneumonia $52(59.1 \%)$ & Klebsiella pneumonia 51 (41.8\%) \\
Mixed 6 (6.8\%) & Streptococcus $4(3.3 \%)$ \\
Escherichia coli 5 (5.7\%) & Bacteroides fragilis 3 (2.5\%) \\
Pseudomona aeruginosa 1 (1.1\%) & Mixed 1 (1\%) \\
Porphymonas 1 (1.1\%) & Escherichia coli 1 (1\%) \\
Salmonella 1 (1.1\%) & Staphylococcus 1 (1\%) \\
Alpha-streptococcus 1 (1.1\%) & Salmonella 1 (1\%) \\
Citrobacter 1 (1.1\%) & Pseudomona aeruginosa 1 (1\%) \\
& Peptostreptococcus 1 (1\%)
\end{tabular}

5.3 days. The PLA patients who need a longer time until a diagnosis has been established usually need surgical intervention, while the earlier PLA is diagnosed, the simpler is the treatment, even antibiotic administration only.

Klebsiella pneumonia remains the major pathogen cultured from the blood $(41.8 \%)$, as well as pus $(59.1 \%)$, in Taiwan. The rate of no growth from blood cultures is higher ( $46.7 \%$ vs. $22.7 \%)$ than from pus. Other commonly seen pathogens are mixed, Escherichia coli, Pseudomonas aeruginosa, Porphymonas, Salmonella, Alpha-Streptococcus, and Citrobacter.

In 2008, Dr. Hope studied the optimal treatment of liver abscesses, and he concluded that if the liver abscess is less than $3 \mathrm{~cm}$ in diameter, parenteral antibiotic administration is suitable therapy, and for those larger than $3 \mathrm{~cm}$ with an uniloculated abscess, also drainage is necessary. Open drainage with surgical intervention is indicated if the abscess is larger than $5 \mathrm{~cm}$ in diameter and multiloculated $[2,3]$. Dr. Ferraioli advised that percutaneous drainage lowered morbidity and is relatively cheaper than surgical intervention [4]. This result is compatible with those of our study showing that parenteral antibiotics plus drainage $(60.3 \%)$ is more commonly used nowadays in the treatment of PLA than surgical intervention (8.7\%).

Seventeen percent of PLA patients went into shock in the clinical course. We found that PLA patients who received surgical interventions showed higher rates of shock in the

Table 3 The mortality rates of four groups of treatment in 126 PLA patients. DFA = days of fever on arrival at the hospital

\begin{tabular}{llll}
\hline $\begin{array}{l}\text { Group } \\
(\mathrm{n}=126)\end{array}$ & Treatment & Mean DFA & Mortality \\
\hline $\mathrm{M}(\mathrm{n}=36)$ & Antibiotic & 3.3 & $2(5.6 \%)$ \\
$\mathrm{MD}(\mathrm{n}=76)$ & Antibiotic + Drainage & 5 & $3(4 \%)$ \\
MS ( $\mathrm{n}=11)$ & Antibiotic + Surgery & 6.5 & $0(0 \%)$ \\
MDS (n=3) & Antibiotic + Drainage + Surgery & 5.3 & $0(0 \%)$ \\
\hline
\end{tabular}

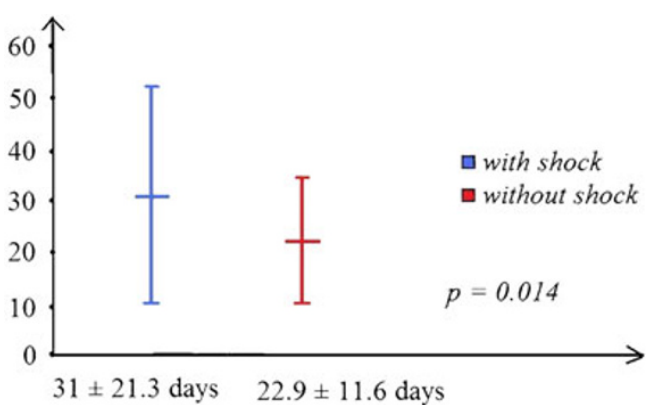

Fig. 2 The PLA patients with shock had significantly longer (8-day) length of stay (LOS) than those without shock

clinical course. The optimal timing of surgical drainage is at the time of shock presentation. The possibility of shock presentation in the surgical treatment group of PLA is 2.5 -fold higher than in the non-surgical treatment group. Shock status, acute renal failure, and acute respiratory failure are the poor prognostic factors of PLA $[5,6]$.

The overall mean hospital stay was 24.2 days. There was obvious prolongation of the hospital stay in shock presentation compared to the non-shock status (31 versus 22.9 days). The current mortality rate is low, ranging from $4.2 \%$ to $11.7 \%[2,7]$. In our study, only $4 \%$ died in the hospital. The groups (MS and MDS) with surgical interventions had no mortality.

\section{Conclusions}

Nowadays we mainly treat pyogenic liver abscesses (PLA) with parenteral antibiotics with drainage. Patients who are treated only with parenteral antibiotics are the ones with early diagnosis of PLA with mean fever duration of 3.3 days and a shorter hospital stay (20.6 days). In PLA patients with shock, a higher rate of surgical intervention is mandatory to save their lives. PLA patients with shock presentation also had 8.1 days longer hospital stays than those who had no shock.

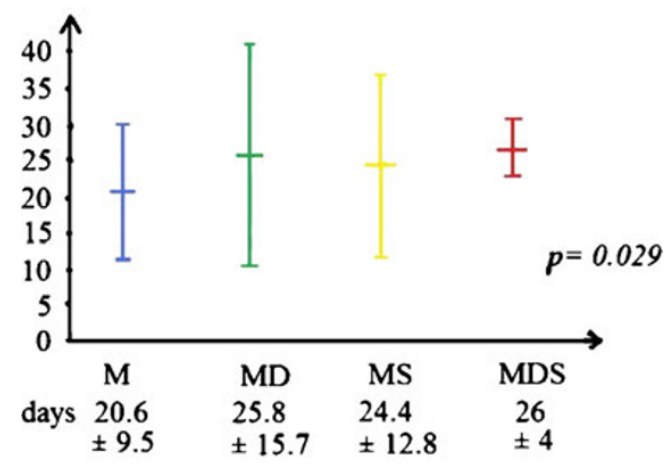

Fig. 3 LOS in four groups of treatment. There was significantly shorter LOS in the group with medical treatment only 


\section{Conflict of interest None}

Open Access This article is distributed under the terms of the Creative Commons Attribution Noncommercial License which permits any noncommercial use, distribution, and reproduction in any medium, provided the original author(s) and source are credited.

\section{References}

1. Chou CY, Lee JJ, Lai YC et al (2008) Successful conservative treatment of a hepatic abscess originating from a ruptured renal abscess in an elderly female. Int J Gernotol 2(3):136-139

2. Hope WW, Vrochides DV, Newcomb WL et al (2008) Optimal treatment of hepatic abscess. Am Surg 74(2):178-182
3. Chung YF, Tan YM, Lui HF et al (2007) Management of pyogenic liver abscesses - percutaneous or open drainage? Singapore Med J 48(12):1158-1165

4. Ferraioli G, Garlaschelli A, Zanaboni D et al (2008) Percutaneous and surgical treatment of pyogenic liver abscesses: observation over a 21-year period in 148 patients. Dig Liver Dis 40(8):690696

5. Chen W, Chen $\mathrm{CH}$, Chiu KL et al (2008) Clinical outcome and prognostic factors of patients with pyogenic liver abscess requiring intensive care. Crit Care Med 36(4):1184-1188

6. Ruiz-Hernández JJ, León-Mazorra M, Conde-Martel A (2007) Pyogenic liver abscesses: mortality-related factors. Eur J Gastroenterol Hepatol 19(10):853-858

7. Lee KT, Wong SR, Sheen PC (2001) Pyogenic liver abscess: an audit of 10 years' experience and analysis of risk factors. Dig Surg 18(6):459-465, discussion 465-6 